\title{
Mitchell Timothy, Petrocratia. La démocratie à l'âge du carbone, Alfortville, Edition Ere, 2011.
}

Bruno Villalba

\section{(2) OpenEdition}

Journals

Édition électronique

URL : http://journals.openedition.org/developpementdurable/9151

DOI : 10.4000/developpementdurable.9151

ISSN : 1772-9971

Éditeur

Association DD\&T

Référence électronique

Bruno Villalba, « Mitchell Timothy, Petrocratia. La démocratie à l'âge du carbone, Alfortville, Edition Ere, 2011. », Développement durable et territoires [En ligne], Vol. 3, n 1 | Mai 2012, mis en ligne le 05 juin 2012, consulté le 22 septembre 2020. URL : http://journals.openedition.org/developpementdurable/ 9151 ; DOI : https://doi.org/10.4000/developpementdurable.9151

Ce document a été généré automatiquement le 22 septembre 2020

Développement Durable et Territoires est mis à disposition selon les termes de la licence Creative Commons Attribution - Pas d'Utilisation Commerciale 4.0 International. 


\section{Mitchell Timothy, Petrocratia. La démocratie à l'âge du carbone, Alfortville, Edition Ere, 2011.}

\section{Bruno Villalba}

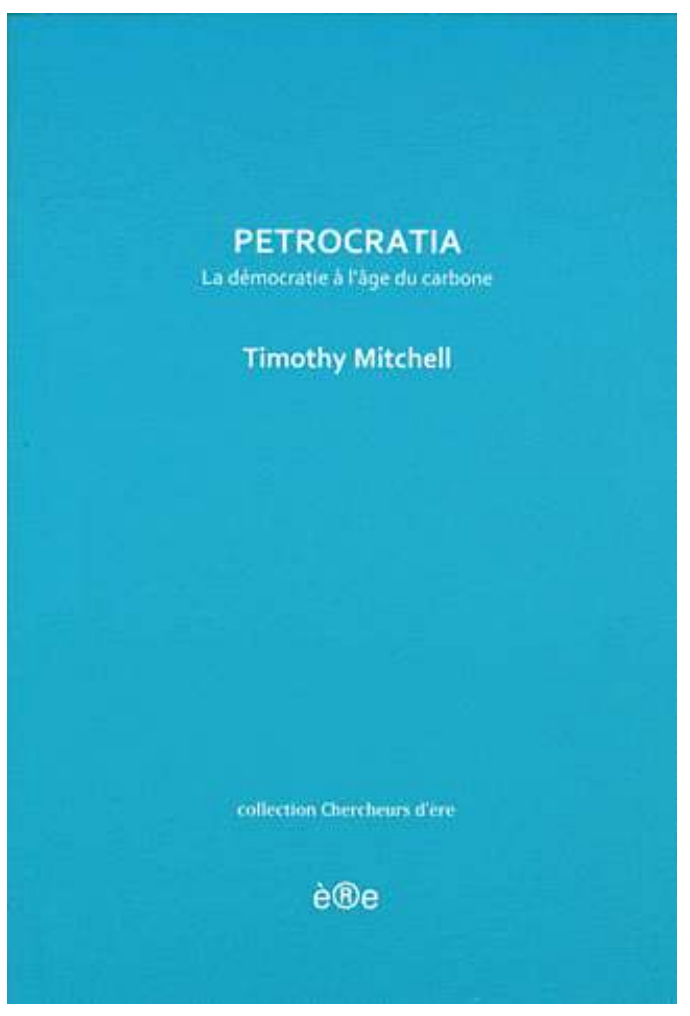

1 Le livre de Timothy Mitchell peut se lire comme une contribution originale à l'histoire de la construction des démocraties modernes ${ }^{1}$. Sa perspective est de montrer comment la politique moderne - celle de la démocratie représentative - ne peut se comprendre, avant tout, sans l'étude de ses soubassements matériels et écologiques : «l'important est de lui restituer sa dimension physico-chimique » (p.13). C'est ce qu'il nomme la Petrocratia. 
2 Depuis des dizaines d'années, de nombreux intellectuels s'interrogent sur les origines, les fondements abstraits de la démocratie représentative. Citons les travaux exemplaires de Bernard Manin, ou les réflexions auto-référencées de Marcel Gauchet ou Pierre Rosanvallon. Ces auteurs poursuivent, de livres en livres, une réflexion sur la construction de la mécanique démocratique, en insistant tour à tour sur l'histoire des idées et sur la construction des pratiques et rituels démocratiques, en insistant aussi sur les crises frappant ce système (crises politiques essentiellement, c'est-à-dire résultant d'un passage d'une pratique démocratique à une autre, par exemple de la démocratie censitaire à représentative). Pourtant, dans ces travaux, malgré leur ampleur et le champ sans cesse étendu de leurs interrogations, la nature demeure, mis à part quelques timides prises en considération, ignorée. Chez ces auteurs, la dimension politique de la matérialité écologique n'existe pas. Elle n'est qu'une simple donnée, externalisée du domaine politique. Dans les travaux de ces auteurs, la nature n'a de valeur politique qu'en tant que potentiel de développement et de puissance économique: on l'envisage alors comme une simple marchandise. Les produits énergétiques ne font pas exception : bien qu'ils soient des éléments indispensables de la puissance politique de la démocratie, leur soutien matériel reste toujours silencieux et anonyme.

3 Mitchell impulse un puissant mouvement de renversement. Il décrit la modernité comme une "démocratie carbone": comme le précise dans sa très stimulante introduction Julien Vincent, « les régimes politiques que nous appelons des démocraties, (...) dépendent et présupposent l'utilisation de ces ressources énergétiques carbonnées comme le charbon et le pétrole (...); ces régimes ne sont donc pas des êtres purement politiques, mais sont des hybrides, encastrés dans un environnement matériel grandement défini par ses ressources énergétiques minérales. » (p. 13).

\section{Une autre série historique}

4 A sa manière, Mitchell participe à la construction d'une histoire politique de la démocratie à partir du postulat selon lequel les conditions matérielles d'existence de cette démocratie sont prépondérantes pour comprendre les conditions sociales de sa construction, ainsi que l'imaginaire politique dont elle se dote. Il ne s'agit pas d'attendre de ce livre, comme l'auteur le signale lui-même, une substitution complète : "suivre la piste du carbone ne signifie pas remplacer les schémas de pensées idéalistes des experts en démocratie par une explication matérialiste, ni réduire les phénomènes politiques à leurs seules causes énergétiques (...) Il s'agit plutôt de partir du constat que le carbone doit être transformé (...) » (p. 21) afin d'assurer par ce travail « la conversion d'une ressource naturelle en une forme de pouvoir » (idem). L'essentiel est donc d'abord de reconnaitre l'existence d'une interaction entre cette ressource et l'organisation politique qu'elle a permis de construire, pour saisir la complexité de ses conséquences, et ainsi être en mesure " d'examiner de près une série de liens entre les combustibles carbonés (charbon, pétrole) et certaines formes de politiques démocratiques ou antidémocratiques» (p. 97). Les ressources naturelles ne sont plus alors conçues comme de simples outils de la construction de la puissance des démocraties modernes. Dès lors, le principe narratif selon lequel l'innovation technique ${ }^{2}$ utilise ces ressources pour produire une richesse que la démocratie se charge de partager ne peut plus fonctionner. 
5 L'étude de cette hybridation (matérialité-idéal politique) occupe la première partie de cet ouvrage. Les chapitres 1 et 2 montrent que l'organisation de la société démocratique - société des masses, de la représentation et de l'abondance matérielle - se constitue à partir du choix de la puissance de l'énergie ${ }^{3}$, mais aussi de sa concentration et de sa reproduction. La politique des masses résulte ainsi de la maitrise d'une technique (la force motrice). L'utilisation du charbon redessine non seulement l'organisation sociale de la production (dissociation de la force physique et de la machine, recomposition du statut du travailleur, etc.), mais aussi le territoire de l'exercice de la démocratie. La ville est le lieu de l'exercice de la démocratie. Or, jusqu'à présent, dépendante du bois pour son énergie, elle était contrainte de s'étaler pour accéder au mieux à cette ressource. Le charbon permet à la ville de se concentrer sur un territoire plus restreint: le développement des transports (réorganisés eux-mêmes par une énergie plus dense et abondante) permet de fournir l'énergie sans être assujettie à un large territoire de contrôle. La ville se regroupe, l'activité politique se concentre... Cela offre à l'action publique la possibilité d'étendre son territoire d'intervention (de la transformation agraire à l'aventure coloniale). Le charbon transforme aussi les rapports sociaux; ainsi, il offre un pouvoir inégalé aux mineurs, qui par leurs actions (grève, sabotage, productivité...) peuvent toucher directement l'ensemble des rouages du pays: "Si donc les grèves devinrent efficaces, ce ne fut pas en vertu de l'isolement des mineurs, mais au contraire parce que le carbone extrait dans les galeries souterraines était relié à chaque usine, chaque bureau, chaque foyer, chaque moyen de transport fonctionnant à la vapeur où à l'électricité.» (p. 35) C'est alors que l'usage du pétrole va contribuer à redéfinir le pouvoir des travailleurs... à la baisse, bien sûr! Mitchell s'appuie sur les propriétés physiques du pétrole pour montrer qu'elles ont façonné un rapport politique et une organisation du travail différents. Produit à l'étranger, il réduit «à néant la capacité des travailleurs à frapper le système de l'énergie dans ses points névralgiques» (p. 60) ${ }^{4}$.

\section{Une économie du carbone}

6 La seconde partie (troisième chapitre) s'interroge principalement sur le rôle des experts (notamment économiques) dans la réalisation de cet évitement matérialiste. Comment la construction de l'expertise économique a pu se construire en dehors de l'évidente matérialité des conditions de création de la richesse? Lorsqu'il examine l'économie du carbone, Mitchell montre comment l'expertise économique a, au XXe siècle, développé un rapport particulier aux hydrocarbures. "L'expertise repose sur la fabrication de mondes maîtrisables » (p. 84) ; autrement dit, le rôle de l'expert économique consiste avant tout à construire une représentation et une utilisation du monde conforme à ce qui est présenté comme scientifiquement souhaitable.

7 Mitchell questionne principalement l'approche keynésienne, dont on ne peut pas dire qu'elle ressorte grandie de cette confrontation (p. 82-89). Cette perception s'éloigne ainsi de la conception antérieure de l'économie qui se fondait sur la compréhension des processus physiques (dimensions spatiales, ressources matérielles... p. 87). Ceux-ci étant limités par nature, l'hypothèse d'une croissance continue ne pouvait être raisonnablement défendue. Le pétrole est, à ce tournant $\mathrm{du} \mathrm{XXI}^{\mathrm{e}}$ siècle, considéré comme abondant et disponible; cela permet à l'idéal de la croissance de devenir le nouvel objectif politique du milieu du XXe. Mieux encore : le pétrole est assimilé au 
dollar, et pèse dans la définition du PIB. La ressource fossile devient désormais l'instrument de la mesure de la réussite productive des sociétés démocratiques. Les économistes de la croissance aboutissent à l'unification « de la démocratie occidentale, du pétrole et du dollar américain. » (p. 78).

\section{Survivre à la disparition?}

8 La thèse de Mitchell aboutit donc, logiquement, à une réflexion sur les limites physiques et politiques d'une telle dépendance à l'égard du pétrole. Les états industrialisés - et ceux qui s'engouffrent dans un tel modèle - sont dépendants de l'or noir. Nous avons développé un mode de vie (consommation, soins, déplacement, logement...) qui nécessite une immense quantité d'énergie, tirée du pétrole et autres combustibles fossiles ${ }^{5}$. Est-il besoin de rappeler que cette quantité est limitée ? Un tel constat fait déjà partie de l'histoire de nos sociétés modernes. Mitchell apporte une réflexion supplémentaire : "Comment la machine politique conçue pour gouverner à l'ère des combustibles fossiles pourrait-elle survivre à leur disparition?» (p. 21) Le pétrole a permis la création et la propagation rapide d'un certain style de vie. Il voit le jour aux États-Unis d'abord, puis dans le reste du monde, par mimétisme plus ou moins choisi. Ce style de vie repose sur une importante consommation d'énergie (p. 65). Au début des années 1970, cette consommation frénétique sera remise en cause, provisoirement, lorsque l'on accepte de reconnaître qu'il existe des limites physiques à cette ressource. Paradoxe d'un système mondial basé sur la production d'une ressource - afin de constituer un régime d'abondance, condition sine qua non pour assurer cette société des égaux que l'on ne cesse de vouloir revendiquer ${ }^{6}$ - et qui toutefois gère la rareté pour en tirer le plus de bénéfice possible...

9 La démonstration s'appuie sur un vaste panorama comparatif. On pourra regretter qu'elle soit parfois rapide dans certains segments. Ainsi, l'auteur évoque à plusieurs reprises l'idée que la rareté du pétrole serait gérée afin de permettre une meilleure efficacité des outils financiers internationaux (régulation monétaire, stratégies spéculatives...), sans toutefois apporter plus d'éléments. Lorsqu'il évoque «le rôle joué par les compagnies pétrolières dans l'ensemble des politiques écologiques» (p. 93), on aimerait en savoir beaucoup plus... Même insatisfaction en ce qui concerne les moyens démocratiques à développer pour contrôler cette relation entre ressource et démocratie (dernière phrase de sa trop rapide conclusion). Il est vrai, que Mitchell présente cet ouvrage comme une contribution à une réflexion plus vaste qui devrait faire l'objet d'une prochaine publication. On pourra cependant se consoler dans l'abondante littérature citée et bien souvent commentée par l'auteur.

10 Ce petit ouvrage (110 pages) est donc extrêmement stimulant! Par son refus de s'extraire des conditions matérielles de construction des régimes démocratiques, Mitchell offre l'opportunité au lecteur de s'interroger sur la fragilité de notre organisation politique. 


\section{NOTES}

1. Timothy Mitchell est à la fois historien, politiste, anthropologue, spécialiste du monde arabe et auteur d'une série de travaux remarqués sur les enjeux géopolitiques du Moyen Orient, ainsi que d'une analyse sur l'expertise. http://www.columbia.edu/ tm2421/ La traduction de l'anglais (US) est de Nicolas Vieillescazes.

2. Cependant, la dimension technique n'est pas suffisamment explorée, voir les réflexions fondatrices de Lewis Mumford, Technics and Civilization, New York, Harcourt Brace and Cie, 1934 (traduit au Seuil en 1976).

3. Voir les analyses d'Alain Gras, Le choix du feu. Aux origines de la crise climatique, Paris, Fayard, 2007.

4. L'introduction rappelle cependant qu'une grève des raffineries est tout aussi efficace...

5. Ce dont fait partie l'uranium: on peut regretter que l'auteur ne prolonge pas un peu sa réflexion sur cette ressource. On pourra cependant la poursuivre à travers le livre remarquable de Gabrielle Hecht, Le rayonnement de la France. Energie nucléaire et identité nationale après la Seconde guerre mondiale, Paris, La Découverte, 2004.

6. Voir Pierre Rosanvallon, La société des égaux, Paris, Le Seuil, coll. Les Livres du Nouveau Monde, 2011. Le livre de Mitchell n'évoque guère les enjeux matériels de l'égalité face aux ressources naturelles... La démocratie demeure un régime extérieur aux contraintes matérielles qui conditionnent cependant son devenir, notamment en ce qui concerne l'égalité.

\section{AUTEUR}

\section{BRUNO VILLALBA}

Bruno Villalba est maître de conférences en science politique à Sciences Po Lille. Il est membre $\mathrm{du}$ Ceraps. Ses travaux portent sur la sociologie de l'environnement. Il est directeur du comité de lecture de la revue DDT. Bruno.villalba[at]sciencespo-lille.eu 